\title{
A NECESSARY AND SUFFICIENT CONDITION FOR UNIFORM APPROXIMATION BY CERTAIN RATIONAL MODULES
}

\section{J. J. CARMONA DOMENECH}

\begin{abstract}
Let $X$ be a compact subset of $\mathbf{C}$ with empty interior and let $g$ be a complex function of class $C^{2}$ in a neighborhood of $X$. For $Z=\{z \in$ $X \mid \partial g(z) / \partial \bar{z}=0\}$, we prove that $R(X)+g R(X)$ is uniformly dense in $C(X)$ if and only if $R(Z)=C(Z)$.
\end{abstract}

If $X$ is a compact subset of $\mathbf{C}$, we denote by $R_{0}(X)$ the algebra of rational functions with poles off $X$, and by $R(X)$ the uniform closure of $R_{0}(X)$ in $C(X)$. We use the symbol $\bar{\partial}$ for the differential operator $\partial / \partial \bar{z}=\frac{1}{2}(\partial / \partial x+i \partial / \partial y)$.

The purpose of this paper is to present a proof of the following result:

THEOREM. Suppose that $X$ is a compact subset of $\mathbf{C}$ with empty interior, and that $g$ is a complex function twice continuously differentiable on a neighborhood of $X$. Write $Z=\{x \in X \mid \bar{\partial} g(z)=0\}$. Then, $R_{0}(X)+g R_{0}(X)$ is uniformly dense in $C(X)$ if and only if $R(Z)=C(Z)$.

COROLlARY. If $X$ is a compact subset of $\mathbf{C}$ with no interior, and if $h$ is an entire function, then $R_{0}(X)+\bar{h} R_{0}(X)$ is uniformly dense in $C(X)$.

The particular case of the above corollary in which $h(z)=z, z \in \mathbf{C}$, was conjectured in O'Farrell [3] and Wang [6], and it has been recently proved by Trent and Wang [5]. Rational modules of type $R_{0}(X)+R_{0}(X) \bar{z}$ had been introduced by O'Farrell [3] in connection with problems of rational approximation in Lipschitz norms.

The basic tool used in the proof of the theorem is an integral formula for infinitely differentiable functions with compact support disjoint from $Z$ (1). In a second step we introduce an integral kernel, associated to $g$, and the corresponding transforms of compactly supported measures. Using this transform, we are able to prove that a measure on $X$, orthogonal to $R_{0}(X)+g R_{0}(X)$ must be supported on $Z$, and then, the desired conclusion follows via standard arguments.

We proceed now to state and prove several results which will be needed in the proof of the theorem.

We denote by $m$ the Lebesgue measure on $\mathbf{C}$, and by $C^{2}(U)$ (resp. $\left.D^{2}(U)\right)$ the set of complex functions (resp. compactly supported functions) twice continuously differentiable on the open subset $U$ of $\mathbf{C}$.

Received by the editors July 21, 1981 and, in revised form, February 2, 1982.

1980 Mathematics Subject Classification. Primary 30A82; Secondary 46J99.

Key words and phrases. Rational module, orthogonal measure. 
Proposition 1. Let $G$ be an open bounded subset of $\mathbf{C}$ with piecewise smooth boundary. Let $U$ be an open set, $U \supset \bar{G}$, and let $f, g \in C^{2}(U)$. Put $S=\{w \in U \mid$ $\bar{\partial} g(w)=0\}$. If $\bar{G} \cap S=\varnothing$, then, for each $w \in G$, we have

$$
\begin{aligned}
f(w)= & \frac{1}{2 \pi i} \int_{\partial G} \frac{f(z)}{z-w} d z-\frac{1}{2 \pi i} \int_{\partial G} \frac{\bar{\partial} f}{\bar{\partial} g}(z) \frac{g(z)-g(w)}{z-w} d z \\
& +\frac{1}{\pi} \int_{G} \bar{\partial}\left(\frac{\bar{\partial} f}{\bar{\partial} g}\right)(z) \frac{g(z)-g(w)}{z-w} d m(z) .
\end{aligned}
$$

Proof. For a fixed $w \in G$, we take $\epsilon>0$ such that $D_{\epsilon}$, the closed disc centered at $w$ with radius $\epsilon$, satisfies $D_{\epsilon} \subset G$. Then $G_{\epsilon}=G-D_{\epsilon}$ is a bounded open set with piecewise smooth boundary. We suppose this boundary endowed with the orientation induced by the usual one in $G_{\epsilon}$. We consider the differential form, of class $C^{1}$ in a neighborhood of $\bar{G}_{\epsilon}$,

$$
\frac{\bar{\partial} f}{\bar{\partial} g}(z) \frac{g(z)-g(w)}{z-w} d z
$$

and we apply Stokes' theorem to obtain

$$
\begin{aligned}
& \int_{G_{\epsilon}} \bar{\partial}\left(\frac{\bar{\partial} f}{\bar{\partial} g}\right)(z) \frac{g(z)-g(w)}{z-w} d \bar{z} \wedge d z+\int_{G_{\epsilon}} \frac{\bar{\partial} f(z)}{z-w} d \bar{z} \wedge d z \\
&=\int_{\partial G} \frac{\bar{\partial} f}{\bar{\partial} g}(z) \frac{g(z)-g(w)}{z-w} d z-\int_{|z-w|=\epsilon} \frac{\bar{\partial} f}{\bar{\partial} g}(z) \frac{g(z)-g(w)}{z-w} d z
\end{aligned}
$$

The last integral is $O(\epsilon)$ because the integrand is bounded uniformly on $G_{\epsilon}$. The integrands of the first two integrals are majorized in modulus by a function of the type $c /|z-w|$, where $c$ does not depend on $\epsilon$. Then, since $1 /|z-w|$ is locally integrable as a function of $z$, letting $\epsilon \rightarrow 0$, we get

$$
\begin{gathered}
\int_{G} \bar{\partial}\left(\frac{\bar{\partial} f}{\bar{\partial} g}\right)(z) \frac{g(z)-g(w)}{z-w} d m(z)+\int_{G} \frac{\bar{\partial} f(z)}{z-w} d m(z) \\
=\frac{1}{2 i} \int_{\partial G} \frac{\bar{\partial} f}{\bar{\partial} g}(z) \frac{g(z)-g(w)}{z-w} d z .
\end{gathered}
$$

Now, to obtain the desired conclusion, it is sufficient to write the second integral of the left hand in terms of $f(w)$ by means of the generalized Cauchy formula [1, p. 151].

COROLlARY 1. Let $g \in C^{2}(\mathbf{C})$ have bounded first derivatives, and put $S=\{w \in$ $\mathbf{C} \mid \bar{\partial} g(w)=0\}$. If $f \in D^{2}(\mathbf{C})$ and $(\operatorname{supp} f) \cap S=\varnothing$, then we have, for each $w \in \mathbf{C}$,

$$
f(w)=\frac{1}{\pi} \int \bar{\partial}\left(\frac{\bar{\partial} f}{\bar{\partial} g}\right)(z) \frac{g(z)-g(w)}{z-w} d m(z) .
$$

PROOF. We distinguish three cases:

(a) $w \notin S$. Let $G$ be a bounded open set with piecewise smooth boundary such that supp $f \subset G, w \in G$ and $\bar{G} \cap S=\varnothing$. We can now apply the above proposition to obtain (1).

(b) $w \in \partial S$. We observe that the integral in (1), as a function of $w$, is continuous. This is because it can be written in the form: $C_{1}+g C_{2}$ where $C_{1}$ and $C_{2}$ are 
convolutions of compactly supported functions with the locally integrable function $1 / z$, and therefore continuous $[1$, p. 154] then (1) follows from (a).

(c) $w \in \stackrel{\circ}{S}$. The integral in (1) is a holomorphic function of $w$ on $\stackrel{\circ}{S}$, because $C_{1}$, $C_{2}$ and $g$ are holomorphic there. Moreover it is bounded (because of the hypothesis on the derivatives of $g$ ) and it vanishes on $\partial \stackrel{S}{S}$ by (b). Then it vanishes identically on $\stackrel{S}{S}[2$, p. 129]. The proof is complete.

DEFINITION. If $g$ is a function of class $C^{1}$ on $\mathrm{C}$, we define the following Borel function:

$$
k(z, w)=\frac{g(z)-g(w)}{z-w}, \quad z, w \in \mathbf{C}, z \neq w,
$$

If $\mu$ is a regular complex Borel measure on $\mathbf{C}$ with compact support, we define

$$
\check{\mu}(w)=\int k(z, w) d \mu(z), \quad w \in \mathbf{C} .
$$

LEMMA 1. Let $\mu$ be a measure on $\mathbf{C}$ with compact support. Then $\check{\mu}$ is well defined on the whole $\mathbf{C}$, it is continuous except possibly on the countable set

$$
A_{\mu}=\{w \in \mathbf{C}|| \mu \mid(\{w\})>0\}
$$

and it is $m$-locally integrable.

Proof. For every $w \in \mathbf{C}$ it is easily seen, using the mean value theorem, that $k(\cdot, w)$ is bounded on the support of $\mu$.

Put $w \notin A_{\mu}$ and take $w_{n} \rightarrow w$. Then $k\left(z, w_{n}\right) \rightarrow k(z, w)$ except possibly for $z=w$. Therefore the above convergence is a.e. $(\mu)$. But the sequence of functions $k\left(\cdot, w_{n}\right)$ is uniformly dominated on $\operatorname{supp} \mu$, appealing again to the mean value theorem. Therefore, $\check{\mu}\left(w_{n}\right) \rightarrow \breve{\mu}(w)$, and hence $\breve{\mu}$ is continuous at $w$.

The local integrability of $\breve{\mu}$ follows from the inequality

$$
|\check{\mu}(w)| \leq\left(\|g\|_{\text {supp } \mu}+|g(w)|\right) \tilde{\mu}(w) \text { a.e. }(m),
$$

where

$$
\tilde{\mu}(w)=\int d|\mu|(z) /|z-w|
$$

and from the fact that $\tilde{\mu}$ is $m$-locally integrable [1, p. 153].

LEMMA 2. Let $\mu$ be a measure on $\mathbf{C}$ with compact support. Let $g \in C^{2}(\mathbf{C})$, and put $S=\{w \in \mathbf{C} \mid \bar{\partial} g(w)=0\}$. If $f \in D^{2}(\mathbf{C})$ and if $\operatorname{supp} f \cap S=\varnothing$, then

$$
\int f d \mu=\frac{1}{\pi} \int \bar{\partial}\left(\frac{\bar{\partial} f}{\bar{\partial} g}\right)(z) \check{\mu}(z) d m(z) .
$$

Proof. Use Corollary 1 and Fubini's Theorem.

LEMMA 3. Let $\mu$ be a measure supported on a compact subset $X$ of $\mathbf{C}$, and let $g$ and $S$ be as in the above lemma. If $\check{\mu}=0$ a.e. $(m)$, then $\mu$ is supported on $X \cap S$.

PRoOF. It follows from Lemma 2, the regularity of $\mu$ and the density of $D^{2}(\mathbf{C}-S)$ on $L^{1}(K, \mu)$.

Proof of THE TheOREM. Assume first that $R(Z)=C(Z)$. Without loss of generality we can suppose that $g \in D^{2}(\mathbf{C})$. Let $\mu$ be a measure on $X$, orthogonal to 
$R_{0}(X)+R_{0}(X) g$. Obviously, if $w \notin X$ then $\check{\mu}(w)=0$. Now, since $X$ has no interior, an application of Lemma 1 shows that $\check{\mu}=0$ a.e. $(m)$. Therefore, using Lemma 3, we conclude that $\mu$ is supported on $Z=X \cap S$. If $Z=\varnothing$, the proof is complete. Otherwise we have to show that $\int f d \mu=0, f \in C(Z)$. Because of the hypothesis it is sufficient to assume $f \in R_{0}(Z)$. Since $X$ has no interior, a small translation of the poles of $f$ allows us to approximate $f$ uniformly on $Z$ by functions in $R_{0}(X)$, and so the conclusion follows because $\mu$ is orthogonal to $R_{0}(X)$.

Suppose now that $R_{0}(X)+g R_{0}(X)$ is dense in $C(X)$. Take $f \in C(Z)$ and consider a continuous extension $\tilde{f}$ of $f$ to $X$. Then $\tilde{f}$ is uniformly approximable on $X$ by functions in $R_{0}(X)+g R_{0}(X)$, so that $f$ is uniformly approximable on $Z$ by restrictions to $Z$ of functions in $R_{0}(X)+g R_{0}(X)$. To complete the proof it is sufficient to show that $g_{\mid Z} \in R(Z)$. This follows from the fact that $g$ is continuously differentiable on a neighborhood of $Z$, from $\bar{\partial} g(z)=0, z \in Z[1, \mathrm{p}$. 160].

REMARKS. (a) As can be easily proved, in the corollary to the Theorem it is sufficient to assume that $h$ is holomorphic on an open neighborhood $U$ of $X$, nonconstant on any component of $U$. Indeed, $\partial \bar{h} / \partial \bar{z}=\overline{\partial h} / \partial z$ and $\partial h / \partial z$ is holomorphic on $U$, and in this case, $Z$ is a finite set.

(b) As another application of our theorem we can consider the case in which $m(Z)=0$. Appealing to the Hartogs-Rosenthal Theorem [4, p. 47] we conclude that, in this situation, $R_{0}(X)+g R_{0}(X)$ is dense in $C(X)$.

(c) We recall that conditions equivalent to $R(Z)=C(Z)$ are known, which involve the notion of analytic capacity [7], so that a necessary and sufficient condition for the density of $R_{0}(X)+g R_{0}(X)$ in $C(X)$ can be stated in terms of analytic capacity.

(d) It is worthwhile noting that if we suppose, as usual, that the compact $X$ is nowhere dense, an analogous proof to that of the main theorem gives that the uniform closure of $R_{0}(X)+g R_{0}(X)$ in $C(X)$ is the uniform algebra $\left\{f \in C(X) \mid f_{\mid Z} \in\right.$ $R(Z)\}$.

ACKNOWLEDGMENTS. The author wishes to express his gratitude to Professor Joan Verdera for valuable conversations and suggestions.

ADDED IN PROOF. After this paper was accepted for publication we received a copy of the work by T. Trent and J. L. Wang, The uniform closure of rational modules, Bull. London Math. Soc. 13 (1981), 415-420, in which a different proof of the theorem is given.

\section{REFERENCES}

1. A. Browder, Introduction to function algebras, Benjamin, New York, 1969.

2. R. Burckel, An introduction to classical complex analysis, Vol. I, Academic Press, New York, 1979.

3. A. O'Farrell, Annihilators of rational modules, J. Funct. Anal. 19 (1975), 373-389.

4. T. W. Gamelin, Uniform algebras, Prentice-Hall, Englewood Cliffs, N.J., 1969.

5. Tavan Trent and James Li-Ming Wang, Uniform approximation by rational modules on nowhere dense sets, Proc. Amer. Math. Soc. 81 (1981), 62-64.

6. James Li-Ming Wang, Approximation by rational modules on nowhere dense sets, Pacific J. Math. 80 (1979), 293-295.

7. L. Zalcman, Rational approximation, Lecture Notes in Math., vol. 50, Springer-Verlag, Berlin and New York, 1968.

Departamento Teoria Funciones, Facultad de Matematicas, Universidad BARCELONA, GRAN Via No. 585, BARCElona (7), Espa ÑA 